\title{
EL SITIO ALERO CUEVAS, PUNA DE SALTA, ARGENTINA: SECUENCIA DE CAMBIO EN ARTEFACTOS LÍTICOS Y RESOLUCIÓN CRONOLÓGICA MACRORREGIONAL DURANTE EL HOLOCENO TEMPRANO Y MEDIO
}

\author{
ALERO CUEVAS SITE, PUNA OF SALTA, ARGENTINA: \\ SEQUENCE OF CHANGE IN LITHIC ARTIFACTS AND \\ MACRO-REGIONAL CHRONOLOGICAL RESOLUTION DURING \\ THE EARLY AND MIDDLE HOLOCENE
}

\author{
Gabriel E. J. López ${ }^{1}$ y Federico Restifo ${ }^{2}$
}

\begin{abstract}
Este trabajo analiza y discute la presencia y/o ausencia de clases de artefactos líticos con potencial tiempo-sensitivo en el sitio Alero Cuevas, Puna de Salta, Noroeste argentino, comparativamente con registro semejante de otras áreas en las tierras altas del norte de Argentina. Se busca contribuir al estudio de la persistencia temporal, la resolución cronológica y la dispersión espacial de estos artefactos en escala macrorregional entre ca. 11.000 y 3.500 a.p. Los resultados del análisis de la evidencia del sitio Alero Cuevas permitieron distinguir clases cronológicamente diagnósticas. Por su alta representación en este sitio y su comparabilidad macrorregional se destacan las puntas triangulares apedunculadas del Holoceno Temprano y los artefactos "Saladillo" sobre forma base de hojas fechados a fines del Holoceno Medio, entre otras clases como puntas lanceoladas y puntas pedunculadas. Estos estudios contribuyen a caracterizar los procesos de cambio que ocurrieron en una escala de largo plazo no solo en el sitio Alero Cuevas sino en distintas áreas de la Puna argentina.
\end{abstract}

Palabras claves: sitio Alero Cuevas, Holoceno, artefactos líticos tiempo-sensitivos, Puna.

This paper analyzes and discusses the presence and/or absence of time-sensitive lithic artifacts at the Alero Cuevas site, Puna of Salta, Northwest of Argentina, in a comparative perspective with similar records of the highlands in northern Argentina. Our aim is to contribute to the study of the temporal persistence, chronological resolution and spatial dispersion of these artifacts in macro-regional scale between ca. 11,000 and 3,500 BP. Evidence from the Alero Cuevas site allowed to distinguish time-sensitive classes. The presence of Early Holocene unstemmed triangular points and "Saladillo" artifacts on blade blanks dated to the Late Middle Holocene, among other classes such as lanceolate points and stemmed points, are highlighted for their high representation at this site and their macro-regional comparability. These studies contribute to characterize the processes of change that occurred in a long-term scale not only at the Alero Cuevas site but also in other areas of the Argentinian Puna.

Key words: Alero Cuevas site, Holocene, time-sensitive lithic artifacts, Puna.

A lo largo de los Andes centro sur se han planteado diversos procesos relacionados con el cambio cultural tanto en escalas locales como macrorregionales (Núñez et al. 2005; Yacobaccio 2001). En especial, se ha destacado la importancia de los artefactos líticos para el estudio de la evolución cultural de largo plazo (Martínez 2003; Núñez 1992). Algunos artefactos pueden considerarse "tiemposensitivos" por su potencial como indicadores cronológicos relativos (Jones y Beck 1992). A nivel teórico, los atributos de los artefactos permiten conformar clases particulares (O’ Brien y Lyman 2000). Entre estos atributos se suele considerar principalmente la morfología, aunque también caracteres métricos y tecnológicos (Hocsman 2006; Hoguin 2014; Martínez 2003). Un caso típico de clases de artefactos tiempo-sensitivos está constituido por las puntas de proyectil (O' Brien y Lyman 2000). Esta particularidad no solo se evidencia en los artefactos líticos sino también en otros materiales arqueológicos (p.ej. clases de cerámica, arte rupestre, etc.). Se considera que

\footnotetext{
1 CONICET, Instituto de Arqueología, FFyL, UBA. 25 de mayo $2173^{\circ}$ piso, Ciudad de Buenos Aires, CP 1002. gabelope@yahoo.com

2 CONICET, Instituto de Investigaciones en Ciencias Sociales y Humanidades (ICSOH). Av. Bolivia 5150. Ciudad de Salta, CP 4400. federicorestifo@gmail.com
} 
el énfasis en artefactos tiempo-sensitivos resulta relevante para el estudio de procesos de cambio cultural en escalas temporales amplias, y a su vez, permite aproximarse a la cronología de contextos sin posibilidad de dataciones absolutas (p.ej. distribuciones de superficie) (Jones y Beck 1992).

Diversas investigaciones en los Andes centro sur han permitido definir una variedad de clases que por sus atributos morfológicos, métricos y/o tecnológicos son distinguibles entre sí y permiten analizar trayectorias de cambio en escalas de largo plazo (Aschero 1984; Capriles et al. 2011; De Souza 2004; Fernández 1983; Hoguin 2014; Klink y Aldenderfer 2005; Núñez et al. 2005; entre otros).
Específicamente, en la Puna argentina algunas clases han sido asignadas satisfactoriamente a distintos momentos de los cazadores-recolectores que ocuparon la región (Cigliano 1962; Fernández 1983; González 1952), pero no todas están asociadas con cronología absoluta.

En este trabajo, se aporta evidencia para el estudio de la persistencia temporal de diversas clases de artefactos líticos, a partir de la secuencia de ocupaciones humanas fechada a lo largo del Holoceno en el sitio Alero Cuevas, localizado en la Puna de Salta, Noroeste de Argentina (Tabla 1, Figura 1). Precisamente, el objetivo de este trabajo es analizar y discutir la presencia y/o ausencia de

Tabla 1. Cronología radiocarbónica del sitio Alero Cuevas. El asterisco corresponde a los fechados nuevos. Radiocarbon chronology of the Alero Cuevas site. The asterisk corresponds to the new dates.

\begin{tabular}{|c|c|c|c|c|}
\hline Sitio & Laboratorio & $\begin{array}{l}\text { Fechado } \\
\text { a.p. }\end{array}$ & Muestra & Referencia \\
\hline $\begin{array}{c}\text { Alero Cuevas } \\
\mathrm{AC} / \mathrm{C} 2 / \mathrm{X}\end{array}$ & AA 66544 & $643 \pm 35$ & Vegetal & López (2013) \\
\hline $\begin{array}{c}\text { Alero Cuevas } \\
\mathrm{AC} / \mathrm{C} 1 / \mathrm{C} 1\end{array}$ & LP- 1671 & $2.020 \pm 60$ & Vegetal & López (2013) \\
\hline $\begin{array}{c}\text { Alero Cuevas } \\
\mathrm{AC} / \mathrm{C} 7 / \mathrm{C} 1\end{array}$ & AA90385 & $2.277 \pm 54$ & Óseo & López (2013) \\
\hline $\begin{array}{c}\text { Alero Cuevas } \\
\mathrm{AC} / \mathrm{C} 2 / \mathrm{F} 2\end{array}$ & LP- 1655 & $4.210 \pm 70$ & Óseo & López (2013) \\
\hline $\begin{array}{c}\text { Alero Cuevas } \\
\mathrm{AC} / \mathrm{C} 7 / \mathrm{F} 2\end{array}$ & AA90383 & $5.106 \pm 68$ & Carbón & López (2013) \\
\hline $\begin{array}{c}\text { Alero Cuevas } \\
\mathrm{AC} / \mathrm{C} 1 / \mathrm{F} 3\end{array}$ & AA 71137 & $6.506 \pm 58$ & Óseo & López (2013) \\
\hline $\begin{array}{c}\text { Alero Cuevas } \\
\mathrm{AC} / \mathrm{C} 2 / \mathrm{F} 3\end{array}$ & LP- 1759 & $6.510 \pm 80$ & Carbón & López (2013) \\
\hline $\begin{array}{c}\text { Alero Cuevas } \\
\text { AC/C8/F3 }\end{array}$ & LP- 3214 & $7.400 \pm 80^{*}$ & Carbón & \\
\hline $\begin{array}{c}\text { Alero Cuevas } \\
\text { AC/C8/ F3 }\end{array}$ & LP- 3220 & $7.500 \pm 160 *$ & Carbón & \\
\hline $\begin{array}{c}\text { Alero Cuevas } \\
\mathrm{AC} / \mathrm{C} 2 / \mathrm{F} 4\end{array}$ & AA71135 & $8.504 \pm 52$ & Carbón & López (2013) \\
\hline $\begin{array}{l}\text { Alero Cuevas } \\
\text { AC-C2/ F4 }\end{array}$ & AA 71136 & $8.838 \pm 52$ & Carbón & López (2013) \\
\hline $\begin{array}{c}\text { Alero Cuevas } \\
\text { AC- C8/ F4 }\end{array}$ & LP- 3226 & $9.280 \pm 140^{*}$ & Carbón & \\
\hline $\begin{array}{l}\text { Alero Cuevas } \\
\text { AC- C4/ F4 }\end{array}$ & LP- 1736 & $9.650 \pm 100$ & Carbón & López (2013) \\
\hline $\begin{array}{l}\text { Alero Cuevas } \\
\text { AC- C8/ F4 }\end{array}$ & LP- 3236 & $9.880 \pm 100^{*}$ & Carbón & \\
\hline
\end{tabular}




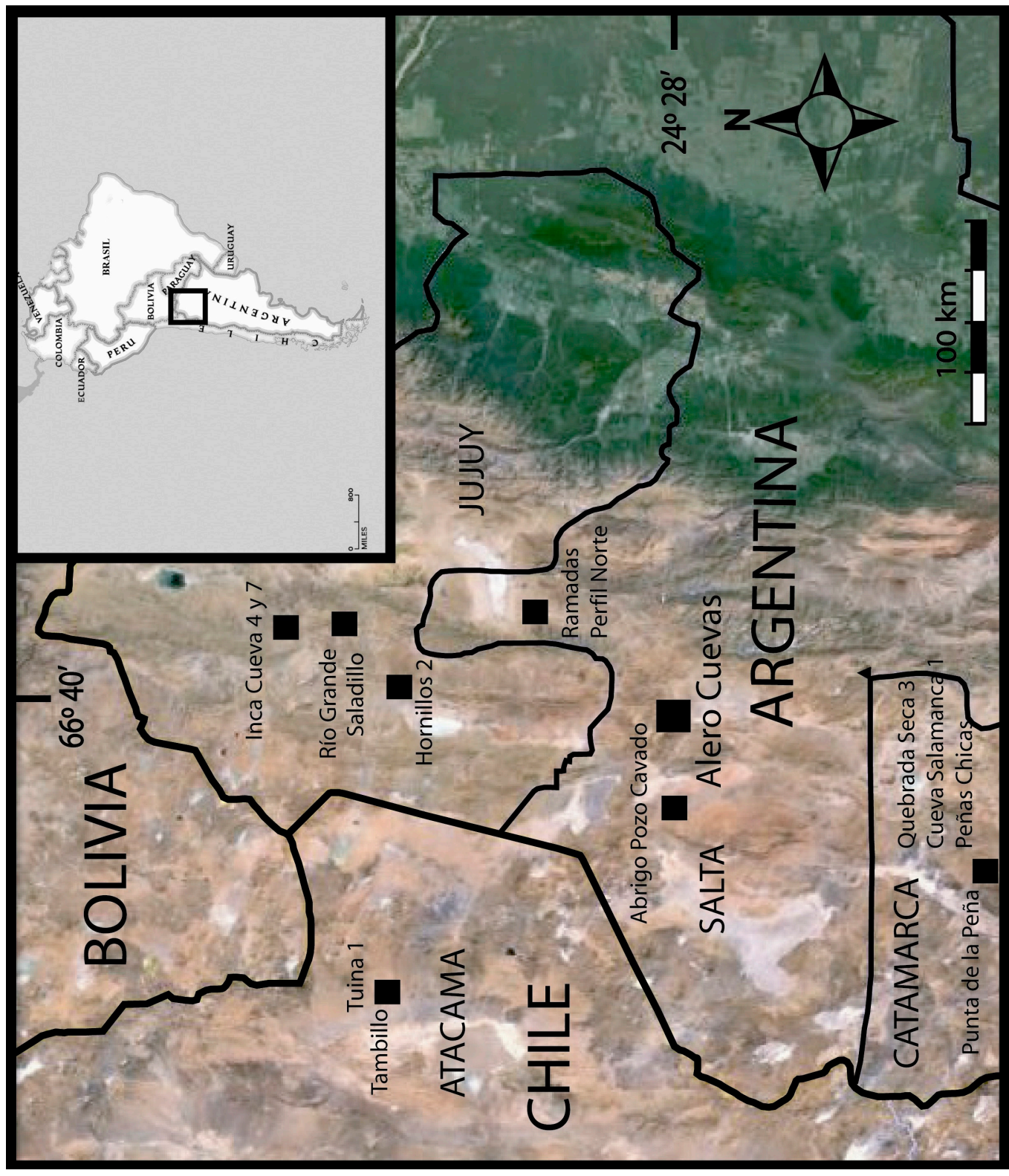


clases artefactuales con potencial tiempo-sensitivo en el sitio Alero Cuevas. Más generalmente, se comparan estas clases con aquellas presentadas por distintos investigadores en sitios de otras áreas de la Puna argentina. La comparabilidad macrorregional permite afinar la resolución cronológica y la persistencia temporal de estos artefactos y discutir su potencialidad como indicadores cronológicamente diagnósticos. A su vez, permite caracterizar la variabilidad espacial en su distribución a partir de la presencia o ausencia de las distintas clases. En consecuencia, estos estudios contribuyen en última instancia a caracterizar los procesos de cambio que ocurrieron en una escala de largo plazo no solo en el sitio Alero Cuevas sino en distintas áreas de la Puna argentina. El lapso de estudio abarca desde las primeras ocupaciones cazadoras recolectoras de la región hasta momentos de cambio hacia nichos económicos de producción de alimentos (ca. 11.000 a.p. - 3.500 a.p.).

\section{Clases Artefactuales Tiempo-sensitivas: Aspectos Teórico-Metodológicos}

Desde un marco evolutivo darwiniano (Boyd y Richerson 1985), se propone que determinadas clases artefactuales surgen como innovaciones y persisten en el tiempo por procesos de transmisión cultural (Bettinger y Eerkens 1999). La persistencia en determinados lapsos dota a estas clases de artefactos de valor tiempo-sensitivo, permitiendo analizar cambios a partir de continuidades y discontinuidades. La variación en la amplitud de los rangos temporales de estas clases permite aproximar cronologías relativas.

En este trabajo, se toma al artefacto como la unidad básica de análisis, focalizando en distintos atributos, los cuales permiten conformar clases particulares. Las clases de artefactos son unidades ideacionales, es decir, construidas por el investigador para medir la variación (Dunnell 1977). En este sentido, a lo largo de las investigaciones en los Andes centro sur, se han construido distintas clases artefactuales con potencial tiempo-sensitivo. Entre ellas, se destacan distintas variantes de puntas de proyectil. Estas clases han servido de base para la construcción de unidades teóricas de mayor inclusividad como industrias o fases (Schobinger 1988). Desde un marco evolutivo darwiniano (Bettinger y Eerkens 1999), se considera que unidades tan inclusivas corren el riesgo de esencializar la variabilidad de datos disponibles dado que tienden a homogeneizar (Clarckson 2005; Muscio 2004; O'Brien y Lyman 2002). Esto porque una perspectiva evolutiva como la que se considera aquí, sostiene una postura ontológica materialista, a partir de la cual la variación es continua, pero analíticamente puede ser ordenada en base a segmentaciones como las clases (Dunnell 1977). Recientemente, se ha comenzado a discutir la confiabilidad de determinados artefactos como indicadores cronológicos (p.ej. Méndez et al. 2015). Se espera que este trabajo contribuya en esa dirección.

Las clases artefactuales con potencial cronológico en el sitio Alero Cuevas se construyeron a partir de las diferencias en los atributos considerados en cada artefacto, independientemente de su grado de recurrencia (Tabla 2). Esta propuesta se acerca a una lógica paradigmática de clasificación, al contrario de una lógica tipológica. Esta última, en un sentido estricto, construye unidades basándose en las tendencias centrales (atributos de mayor recurrencia), mientras que la clasificación paradigmática construye unidades simplemente a partir de la intersección de estados de los atributos, donde todos ellos tienen la misma relevancia para la construcción de clases, sin considerar su frecuencia y el número de especímenes (O’Brien y Lyman 2000). La especificación de atributos y sus variantes depende del interés analítico del investigador (O'Brien y Lyman 2000). En este caso, se seleccionaron atributos y distintas variantes (estados de los atributos) que permitieron la comparación macrorregional con otras clases definidas previamente en la bibliografía (Aschero 1984; Aschero et al. 2011; De Souza 2004; Fernández 1983; Hocsman 2006; Hoguin 2014; López 2013; López y Restifo 2014; Martínez 2003; Moreno 2011; Núñez et al. 2005; Patané Aráoz 2013; Pintar 2014; Ratto 2006; Restifo 2013a; Yacobaccio y Morales 2011; entre otros). La comparabilidad es importante para ajustar la resolución cronológica de distintas clases definidas en la Puna argentina en el rango temporal que abarca este trabajo (ca. 11.000-3.500 años a.p.).

La selección de atributos para la determinación de clases en el sitio Alero Cuevas se realizó bajo la guía general de la propuesta de clasificación de Aschero (1975, 1983), a la vez que se integraron otras propuestas (Bar-Yosef y Kuhn 1999). En este sentido, es importante destacar que la distinción de las variantes de cada atributo se confeccionó apelando a categorías inclusivas, con el objetivo 
Tabla 2. Descripción de las Clases Artefactuales con potencial Tiempo-sensitivo del sitio Alero Cuevas.

$\mathrm{El}$ asterisco indica la medición de un solo espécimen.

La imagen de la clase 7 muestra la cara dorsal de un artefacto (izquierda) y la cara plana en otro (derecha).

Description of artefactual classes with time-sensitive potential at Alero Cuevas site.

The asterisk indicates that we measure only one specimen.

The image of class 7 shows the dorsal face of one artifact (left) and the flat face on another (right).

\begin{tabular}{|c|c|c|c|c|c|c|c|}
\hline Clase & Imagen & $\mathrm{N}$ & $\begin{array}{l}\text { Fechas } \\
\text { (a.p.) }\end{array}$ & Morfología & $\begin{array}{c}\text { Forma } \\
\text { base }\end{array}$ & $\begin{array}{l}\text { Medidas } \\
(\mathrm{mm})\end{array}$ & $\begin{array}{c}\text { Otros } \\
\text { atributos }\end{array}$ \\
\hline Clase 1 & & 11 & $9880-8500$ & $\begin{array}{l}\text { Punta de limbo } \\
\text { triangular, } \\
\text { apedunculada } \\
\text { y de base } \\
\text { recta. }\end{array}$ & Lasca & $\begin{array}{c}\text { Longitud max/min: } 31,6 / 21,5 \\
\text { Ancho max/min: } 23,6 / 17,8 \\
\text { Espesor max/min: } 6,8 / 3\end{array}$ & $\begin{array}{l}\text { Tamaño pequeño, } \\
\text { sección biconvexa, } \\
\text { talla bifacial. }\end{array}$ \\
\hline Clase 2 & & 1 & $9880-8500$ & $\begin{array}{l}\text { Punta de limbo } \\
\text { triangular, } \\
\text { apedunculada } \\
\text { y de base } \\
\text { cóncava } \\
\text { atenuada. }\end{array}$ & Lasca & $\begin{array}{l}\text { Longitud max/min: } 20 \\
\text { Ancho max/min: } 21 \\
\text { Espesor max/min: } 4\end{array}$ & $\begin{array}{l}\text { Tamaño pequeño, } \\
\text { sección biconvexa, } \\
\text { talla bifacial. }\end{array}$ \\
\hline Clase 3 & & 3 & $7500-6500$ & $\begin{array}{l}\text { Punta de limbo } \\
\text { lanceolado, } \\
\text { apedunculada } \\
\text { y de base } \\
\text { convexa }\end{array}$ & Lasca & $\begin{array}{l}\text { Longitud } \max / \mathrm{min}: 57 * \\
\text { Ancho } \mathrm{max} / \mathrm{min}: 28 / 20 \\
\text { Espesor } \max / \mathrm{min}: 10 / 6,5\end{array}$ & $\begin{array}{l}\text { Tamaño grande, } \\
\text { sección biconvexa, } \\
\text { talla bifacial. }\end{array}$ \\
\hline Clase 4 & & 1 & $7500-6500$ & $\begin{array}{l}\text { Punta de limbo } \\
\text { triangular, } \\
\text { pedúnculo } \\
\text { y de base } \\
\text { convexa. }\end{array}$ & Lasca & $\begin{array}{c}\text { Longitud } \mathrm{max} / \mathrm{min}: 29,8 \\
\text { Ancho } \mathrm{max} / \mathrm{min}: 23 \\
\text { Espesor } \mathrm{max} / \mathrm{min}: 7\end{array}$ & $\begin{array}{l}\text { Tamaño pequeño, } \\
\text { sección biconvexa, } \\
\text { talla bifacial. }\end{array}$ \\
\hline Clase 5 & & 1 & $7500-6500$ & $\begin{array}{c}\text { Punta de limbo } \\
\text { triangular, } \\
\text { pedúnculo } \\
\text { destacado } \\
\text { de bordes } \\
\text { convergentes, } \\
\text { hombros y } \\
\text { base recta. }\end{array}$ & Lasca & $\begin{array}{l}\text { Longitud max/min: - } \\
\text { Ancho max/min: } 33,5 \\
\text { Espesor max/min: } 13,5\end{array}$ & $\begin{array}{l}\text { Tamaño grande } \\
\text { (por proyección), } \\
\text { sección biconvexa, } \\
\text { talla bifacial. }\end{array}$ \\
\hline Clase 6 & & 1 & $5100-4200$ & $\begin{array}{l}\text { Punta de limbo } \\
\text { lanceolado, } \\
\text { apedunculada } \\
\text { y de base } \\
\text { convexa. }\end{array}$ & Lasca & $\begin{array}{l}\text { Longitud max/min: } 23 \\
\text { Ancho } \mathrm{max} / \mathrm{min}: 15,5 \\
\text { Espesor } \mathrm{max} / \mathrm{min}: 6\end{array}$ & $\begin{array}{l}\text { Tamaño pequeño, } \\
\text { sección biconvexa, } \\
\text { talla bifacial. }\end{array}$ \\
\hline Clase 7 & & 11 & $5100-4200$ & $\begin{array}{l}\text { Artefacto } \\
\text { formatizado } \\
\text { de limbo } \\
\text { lanceolado, } \\
\text { apedunculado } \\
\text { y base } \\
\text { convexa. }\end{array}$ & Hoja & $\begin{array}{c}\text { Longitud max/min: } 73 / 54 \\
\text { Ancho max/min: } 24 / 19,6 \\
\text { Espesor max/min: } 14,8 / 7,7\end{array}$ & $\begin{array}{c}\text { Tamaño } \\
\text { grande, sección } \\
\text { planoconvexa, talla } \\
\text { predominantemente } \\
\text { unifacial. }\end{array}$ \\
\hline Clase 8 & & 1 & $5100-4200$ & $\begin{array}{l}\text { Punta de limbo } \\
\text { lanceolado, } \\
\text { apedunculada } \\
\text { y de base } \\
\text { recta. }\end{array}$ & Lasca & $\begin{array}{l}\text { Longitud } \max / \mathrm{min}:- \\
\text { Ancho } \mathrm{max} / \mathrm{min}: 22,7 \\
\text { Espesor } \max / \min : 9,9\end{array}$ & $\begin{array}{l}\text { Tamaño } \\
\text { indeterminado, } \\
\text { sección biconvexa, } \\
\text { talla bifacial. }\end{array}$ \\
\hline
\end{tabular}


de facilitar la comparación macrorregional. Los atributos seleccionados fueron los siguientes: forma perimetral del limbo, forma de la base, presencia/ ausencia de pedúnculo y aletas, tipo de forma base, sección transversal, situación de los lascados sobre las caras y tamaño.

El listado de atributos y sus respectivas variantes se constituyó de la siguiente manera:

- Forma perimetral del limbo: Triangular, lanceolada, cordiforme.

- Forma de la base: Recta, convexa, acuminada, cóncava atenuada, cóncava profunda o "escotada".

- Presencia/ausencia de pedúnculo y aletas: Se contempló principalmente la identificación o no de pedúnculo y aletas, y en caso de estar presentes se procedió a su descripción.

- Tipo de forma base: Se utilizó la distinción general entre lascas y hojas. Siguiendo a diferentes autores, se definen a las hojas como piezas de morfología alargada, en las que la longitud representa el doble del ancho o más (Bar-Yosef y Kuhn 1999), y se destaca la presencia de bordes rectos paralelos o subparalelos, y de aristas rectas paralelas o subparalelas en cara dorsal, que remiten a una técnica de talla en particular (Aschero 1975).

- Sección transversal: biconvexa, planoconvexa, triangular, romboidal e irregular.

- Situación de los lascados sobre las caras: unifacial y bifacial.

- Tamaño: Se agruparon en dos variantes: tamaños pequeños y tamaños grandes. La primera agrupa a los tamaños muy pequeños a mediano pequeños, y la segunda, los mediano grandes a muy grandes de Aschero (1983). Esta inclusividad obedece al hecho de que no siempre se observa el empleo de categorías de tamaño con el grado de detalle especificado en la propuesta de clasificación seguida (Aschero 1975, 1983).

Por otra parte, cuando el número de ejemplares lo permitió se determinó la media, el desvío estándar, y el coeficiente de variación de las medidas de ancho, espesor y longitud máximos de los artefactos enteros con potencial tiempo-sensitivo en el sitio Alero Cuevas. Estos análisis fueron posibles en las dos clases con mayor número de especímenes (ver más adelante clases 1 y 7). Los resultados se compararon con aquellos provenientes de diversos sitios estratificados de la Puna argentina (ver más adelante) en los que estas dos clases fueron detectadas. Los indicadores métricos apuntan a discutir la variabilidad regional.

Por último, se destaca que la información del sitio Alero Cuevas se presenta agrupada según las capas en las que se encuentran los materiales, mientras que las clases de artefactos fueron numeradas con el objetivo de ordenarlas y sin ninguna otra connotación.

\section{El Sitio Alero Cuevas en el Contexto Regional}

Este sitio se ubica en la cuenca de Pastos Grandes, en la Puna de Salta (Figura 1). En particular, se localiza en un sector de quebradas de altura por encima de los $4.400 \mathrm{msm}$. La quebrada en la que se encuentra el sitio Alero Cuevas es apta para la ocupación humana dada la existencia de agua permanente proveniente del deshielo de los cerros que la rodean (López 2008). Específicamente, se trata de un abrigo rocoso sobre el talud de un cerro, por encima de una vega con abundante pastura. En este sector actualmente se registran camélidos silvestres como vicuñas (Vicugna vicugna) y guanacos (Lama guanicoe), los recursos de mayor rendimiento en la región. En general, la Puna de Salta es un ambiente de baja productividad primaria, alta heterogeneidad en la distribución de nutrientes y sequías impredecibles, constituyendo un ambiente de alto riesgo (Muscio 2004). Pastos Grandes responde a estas características, aunque hay sectores particulares del espacio con mayor productividad (p.ej. las quebradas).

El sitio Alero Cuevas fue detectado en la campaña de abril de 2004 y comenzó a ser excavado en marzo del año siguiente (López 2008). La roca del alero es una toba dacítica que sobresale en el paisaje de la quebrada por sus grandes dimensiones: un frente de 19,3 m en línea recta y más de $8 \mathrm{~m}$ a la línea de goteo en la parte más profunda. Se distinguieron distintas capas con muy buena preservación de materiales arqueológicos orgánicos e inorgánicos, con una secuencia cronológica en el Holoceno Temprano, Medio y Tardío (López 2008). En este trabajo, se presentan cuatro nuevas fechas radiocarbónicas correspondientes al Holoceno Temprano y Medio, las cuales se suman a otras diez disponibles para distintos momentos del Holoceno (Tabla 1 y López 
2013). Hasta el momento se excavaron $10,5 \mathrm{~m}^{2}$ en distintos sectores del alero, alcanzando una potencia promedio de un metro aproximadamente.

Las capas de las cuales proviene el material analizado corresponden al Holoceno Temprano y Medio (Figura 2). Se trata de las capas F4 (ca. 9.880-8.500 a.p.), F3 (ca. 7.500-6.500 a.p.) y F2 (5.100-4.200 a.p.). Desde una perspectiva general, el Holoceno Temprano en la Puna se caracterizó por un ambiente más húmedo que el actual y una alta movilidad residencial de los grupos humanos (Yacobaccio y Vilá 2002). El Holoceno Medio fue más seco en términos generales, lo cual segmentó el ambiente en parches de recursos específicos y llevó a una concentración de los grupos en esos espacios disponibles (Morales 2011; Yacobaccio y Vilá 2002). Durante el Holoceno Medio, se evidencia un aumento de la diversidad de puntas a nivel macrorregional asociadas con nuevos sistemas de armas y técnicas de caza (Aschero y Martínez 2001; Moreno 2011; Ratto 2006; Restifo 2013b; entre otros). Específicamente, se han propuesto técnicas de caza colectiva de camélidos en un contexto de mayor agrupamiento humano y la utilización de un sistema de armas de lanza arrojadiza. Al contrario, durante el Holoceno Temprano habrían predominado los propulsores de dardo con una técnica de caza de camélidos a distancia y en espacios abiertos (Aschero y Martínez 2001; Moreno 2011; Ratto 2006; Restifo 2013b).

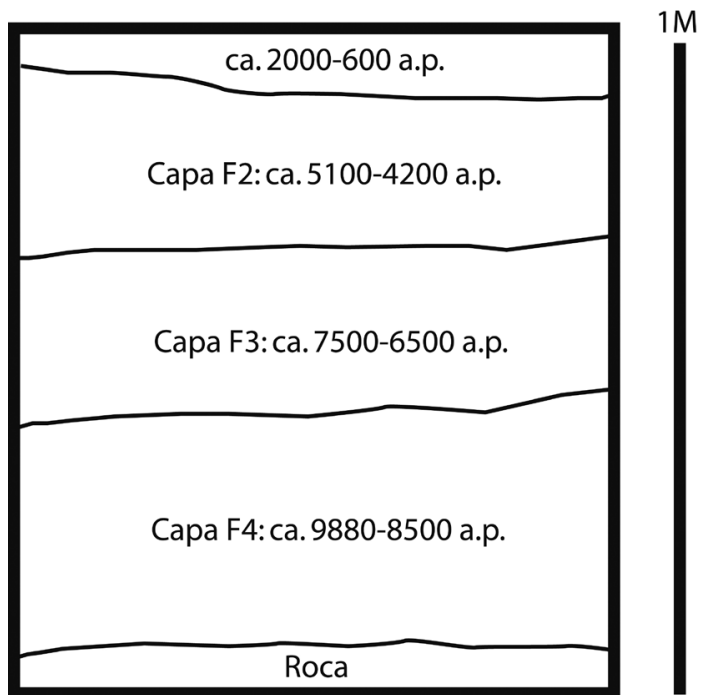

Figura 2. Perfil estratigráfico de la cuadrícula 8 del sitio Alero Cuevas.

Stratigraphic profile of grid 8 at Alero Cuevas site.
El objetivo de este trabajo se centra en las clases con potencial tiempo-sensitivo y por lo tanto no se describen en detalle los materiales arqueológicos de cada capa (López 2008, 2013; Restifo 2013a). Igualmente es pertinente destacar que en toda la estratigrafía la fauna muestra una alta representación de los camélidos sobre otros taxones, mientras que el material lítico no solo indica cambios en las clases de artefactos con potencial tiempo-sensitivo (ver más adelante) sino también una predominancia de obsidianas provenientes de fuentes localizadas entre 40 y 150 km de distancia (Quirón, Archibarca, Zapaleri) y andesitas locales entre las materias primas (López 2008, 2013; Restifo 2013a).

\section{Resultados}

\section{Alero Cuevas capa F4 ca. 9.880-8.500 a.p.}

\section{Clase 1}

Se trata de una punta de proyectil de limbo triangular y apedunculada de base recta con amplia dispersión espacial durante el Holoceno Temprano (Tabla 2). En el sitio Alero Cuevas se recuperaron 11 especímenes de esta clase, de los cuales 7 están enteros y 4 fragmentados. La materia prima predominante es la obsidiana proveniente de Quirón, una fuente ubicada a $40 \mathrm{~km}$ aproximadamente de Alero Cuevas (López 2008; Restifo 2013 a). La morfología triangular presenta leves variaciones en los bordes del limbo, en algunos casos rectos y en otros convexos. La sección es biconvexa. Por su parte, las caras de las piezas exhiben talla bifacial con un gradiente de trabajo que va desde el retoque marginal en ambas caras hacia el retoque bifacial extendido, incluyendo casos intermedios entre ambos extremos. En relación con la forma base, las piezas con menor grado de formatización permitieron establecer el uso de lascas. La Tabla 3 considera la media, el desvío estándar y el coeficiente de variación $(\mathrm{CV})$ de la longitud, el ancho y el espesor máximos de las puntas triangulares apedunculadas del sitio Alero Cuevas, en comparación con la misma clase de puntas procedentes de otros sitios de la Puna argentina como Inca Cueva 4, Hornillos 2 y Huachichocana III en Jujuy, y Quebrada Seca 3, en Catamarca (ver discusión más adelante). Al contrario de Alero Cuevas, en Inca Cueva 4, las materias primas predominantes en las puntas son cuarcitas, basaltos y sílices, al igual que en Huachichocana III 
Tabla 3. Comparación de la media, el desvío estándar (DS) y el coeficiente de variación (CV) de la longitud, ancho y espesor máximos de las clases de artefactos 1 y 7 de Alero Cuevas y otros sitios de la Puna argentina.

Comparison of the mean, the standard deviation $(S D)$ and the coefficient of variation $(C V)$ of the maximun length, width and thickness of artifacts classes 1 and 7 at Alero Cuevas and other sites in the Argentinian Puna.

\begin{tabular}{|c|c|c|c|c|c|c|c|c|c|c|c|}
\hline \multirow{2}{*}{ Clase } & \multirow{2}{*}{ Sitio } & \multirow{2}{*}{$\mathrm{N}$} & \multicolumn{3}{|c|}{ Longitud máxima } & \multicolumn{3}{|c|}{ Ancho máximo } & \multicolumn{3}{|c|}{ Espesor máximo } \\
\hline & & & Media & DS & $\mathrm{CV} \%$ & Media & DS & $\mathrm{CV} \%$ & Media & DS & $\mathrm{CV} \%$ \\
\hline \multirow{5}{*}{1} & Alero Cuevas & 11 & 27,3 & 3,7 & 13 & 21,7 & 2,5 & 11 & 4,8 & 1,06 & 22 \\
\hline & Inca Cueva 4 & 13 & 26,3 & 4,4 & 16 & 20,02 & 2,5 & 12 & 5,1 & 1,3 & 25 \\
\hline & Quebrada Seca 3 & 5 & 33,8 & 10,1 & 29 & 22,9 & 3,2 & 13 & 5,7 & 0,7 & 12 \\
\hline & Hornillos 2 & 4 & 25,29 & 4,43 & 17 & 18,9 & 3,4 & 18 & 5,7 & 0,3 & 5 \\
\hline & Huachichocana III & 7 & 32 & 9,1 & 28 & 20 & 1 & 5 & 5,5 & 2 & 36 \\
\hline \multirow{3}{*}{7} & Alero Cuevas & 11 & 61,9 & 6,2 & 10 & 22,2 & 2,6 & 11 & 9,6 & 2,1 & 21 \\
\hline & Río Grande & 24 & 58 & 10,7 & 18 & 22,2 & 2,3 & 10 & 9,8 & 1,3 & 13 \\
\hline & Ramadas PN & 6 & 66,6 & 2,3 & 3 & 25,2 & 3,2 & 12 & 9,6 & 1,6 & 16 \\
\hline
\end{tabular}

(predominio de cuarcitas) (Aschero 1984; Fernández Distel 1974). En Hornillos 2 y Quebrada Seca 3 predominan las obsidianas (Hoguin 2014; Martínez 2003), casos similares al de Alero Cuevas.

\section{Clase 2}

Se trata de una pieza de limbo triangular, apedunculada, y con la particularidad de presentar una base cóncava atenuada (sensu Aschero 1983). A nivel cronológico, su presencia en la Puna argentina parece acotada al Holoceno Temprano. En este caso particular, es relevante destacar la evidencia de reciclaje hacia un artefacto burilante, probablemente por motivo de fractura, en lo que fuera el ápice de la punta.

La forma base correspondería a lasca. El tamaño es pequeño. Entre los atributos de este espécimen se observa una sección biconvexa y talla bifacial (retoque extendido y parcialmente extendido) (Tabla 2).

\section{Alero Cuevas capa F3, ca. 7.500-6.500 a.p.}

\section{Clase 3}

Por otra parte, en la capa F3 del sitio Alero Cuevas se registraron tres puntas lanceoladas bifaciales, dos fracturadas y una entera (Tabla 2). Esta última, presenta un retoque parcialmente extendido y un grado de formatización que podría indicar que se trata de una preforma de punta, pero tiene todos los atributos de las lanceoladas bifaciales descritas para esta clase, incluyendo una base convexa. Asimismo se destaca un tamaño grande para este ejemplar (Tabla 2). Los artefactos fragmentados tienen retoque bifacial extendido. Potencialmente, se confeccionaron sobre lascas.

\section{Clase 4}

En la capa F3, también se registró un ejemplar de punta de limbo triangular con pedúnculo y base levemente convexa (Tabla 2). Este artefacto se encuentra confeccionado sobre forma base posiblemente de lascas y el tamaño es pequeño (Tabla 2). Presenta una sección biconvexa y trabajo bifacial (retoque parcialmente extendido en una cara y extendido en la otra).

\section{Clase 5}

Se trata de una punta de proyectil registrada en contextos de superficie en distintos ámbitos de la Puna. Su hallazgo en estratigrafía en el sitio Alero Cuevas constituye una novedad en relación con su temporalidad. Se hace referencia a la denominada por Jorge Fernández (1971) como "punta del diablo", la cual presenta un limbo triangular, bordes denticulados, hombros y un pedúnculo de bordes convergentes hacia una base recta (forma trapezoidal) (Tabla 2). El espécimen recuperado en el sitio Alero Cuevas está fragmentado en parte del limbo (Tabla 2). Asimismo, se encontró asociado a un sector de fogón, fechado recientemente en ca. 7.500 a.p. (Tabla 1). Esta fecha marca la posibilidad 
de ubicar cronológicamente una clase de punta de proyectil que tiene antecedentes en la bibliografía (Fernández 1971; Schobinger 1988). La forma base posiblemente corresponde a lasca, y el tamaño, dado que está fragmentado, no es posible calcularlo certeramente. Sin embargo, se estima que superaría los $70 \mathrm{~mm}$. Esto se plantea sobre la base de la medición de un ejemplar entero proveniente de una colección en San Antonio de los Cobres en la Puna de Salta, que con el limbo reactivado alcanza una longitud de $73 \mathrm{~mm}$ (Restifo comunicación personal 2016). Asimismo, la proyección gráfica de los bordes rectos de la punta hallada en Alero Cuevas, sugiere una longitud aún mayor. El ancho es $33,5 \mathrm{~mm}$ y el espesor es de $13,5 \mathrm{~mm}$.

\section{Alero Cuevas capa F2, ca. 5.100-4.200 a.p.}

\section{Clase 6}

En la capa F2, se recuperó un espécimen entero de punta de proyectil lanceolada bifacial y apedunculada (Tabla 2). Corresponde a las lanceoladas pequeñas de base convexa, posiblemente sobre forma base de lasca. Asimismo, tiene una sección biconvexa y retoque bifacial extendido. Esta punta se diferencia de la clase 3 por el tamaño pequeño $(23 \mathrm{~mm}$ de longitud máxima). La dispersión de esta clase de puntas lanceoladas pequeñas está documentada en distintos sitios de la Puna, especialmente hacia fines del Holoceno Medio (Aschero y Yacobaccio 1999).

\section{Clase 7}

Esta clase se caracteriza por la morfología de limbo lanceolado y apedunculado, y sección planoconvexa. Mayormente son piezas de retoque unifacial (cara dorsal). Se registraron 11 especímenes en Alero Cuevas (seis enteros y cinco fragmentados). En otros sectores de la Puna argentina se las ha definido como "puntas monofaciales" o "puntas Saladillo" (Fernández 1971). Sin embargo, la presencia de filos tallados en sus bordes sugiere funciones de procesamiento. Esto ha llevado a adoptar nuevas terminologías como artefactos lanceolados unifaciales o Saladillo (López 2008; Restifo 2015) o lanceoladas sobre hojas (Hoguin 2014). Asimismo, se observa la recurrencia de reducción del bulbo mediante retoque, lo cual habría estado orientado a disminuir el espesor de este sector de la pieza en la cara plana, probablemente vinculado al enmangue. Las piezas de Alero Cuevas son de tamaño grande (Tabla 2). La comparación con las medidas de especímenes de Río Grande, en la Puna de Jujuy, y Ramadas en la Puna de Salta, permite observar baja variación métrica, principalmente en el ancho y la longitud (Tabla 3). La materia prima de todos los ejemplares registrados en el sitio Alero Cuevas es local (andesita y cuarcita con fuentes en la cuenca de Pastos Grandes).

El rasgo tecnológico característico de estos artefactos es su confección sobre hojas. Este representa un cambio tecnológico regional, aunque no se registra en todas las áreas de la Puna, tal como es el caso de Antofagasta de la Sierra, Catamarca (Aschero y Hocsman 2011). En Alero Cuevas, los artefactos "Saladillo" se registran solamente en la capa F2 asociados con un núcleo de hojas (López 2008, Restifo 2015). Este cambio tecnológico parece circunscribirse a esta capa, dado que en las anteriores y posteriores tanto estos artefactos como los núcleos de hojas están ausentes.

\section{Clase 8}

Se caracteriza por un espécimen de limbo lanceolado de base recta. Dado que está fragmentado no puede saberse su tamaño, aunque se pudo calcular el ancho y el espesor (Tabla 2). La forma base sería lasca. Entre otros atributos presenta talla bifacial y sección biconvexa.

\section{Otras clases no presentes en el sitio Alero Cuevas}

Hasta el momento, el sitio Alero Cuevas presenta al menos ocho clases con potencial tiempo-sensitivo. Sin embargo, hay algunas ausencias que conviene destacar para analizar la variabilidad macrorregional en la distribución de las clases. Para contextos tempranos, es notable la ausencia de especímenes de punta de limbo triangular o lanceolado alargado y base escotada, también denominada en Chile como Huiculunche 2 (De Souza 2004), o puntas de limbo triangular o lanceolado con pedúnculo destacado de bordes rectos (Martínez 2003). En la Puna argentina, la primera clase de puntas se registra en el sitio Hornillos 2, en una capa fechada en ca. 8.200 a.p. (Hoguin 2014), mientras que las 
segundas se registraron en Quebrada Seca 3, fechadas en ca. 9.700 a.p. a ca. 7.700 a.p. y en Punta de la Peña 4, fechadas en ca. 3.600 a.p. (Martínez 2003). Esto muestra una discontinuidad de esta clase que da como resultado una resolución cronológica menos ajustada y en consecuencia un menor valor tiempo-sensitivo.

Por el momento, tampoco se registraron puntas de limbo triangular alargado, pedúnculo destacado de bordes convergentes y aletas (morfología tetragonal), también conocida en Chile como San Martín (Núñez et al. 2005; Hoguin y Yacobaccio 2012) o en la Puna de Jujuy como Perchel (Fernández 1971). Sin embargo, esta clase de puntas fue registrada en colecciones de pobladores locales en distintas áreas de la Puna de Salta. Por lo tanto, no se descarta que la ampliación de la excavación en el sitio Alero Cuevas permita reconocer estas puntas. Las fechas de las puntas "San Martín", se ubican entre ca. 8.200 a.p. en Chile (Núñez et al 2005), y ca. 7.400 a.p. en la Puna argentina, más precisamente en el sitio Hornillos 2 (Hoguin y Yacobaccio 2012).

Otras clases ausentes en el sitio Alero Cuevas comprenden una punta de limbo lanceolado, con presencia de denticulado, bordes paralelos rectos, base escotada y tamaño grande, conocida en la Puna de Catamarca como Quebrada Seca C (Martínez 2003), y una punta de limbo lanceolado y tamaño pequeño (tipo mandorla) (Aschero et al. 2011). La primera está presente en el sitio Quebrada Seca 3, Puna de Catamarca con fechas entre ca. 7.100 y 6.500 a.p. (Martínez 2003) y la segunda se evidencia en sitios como Inca Cueva 7, en la Puna de Jujuy (Aschero et al. 2011), y Abrigo Pozo Cavado, en la Puna de Salta (López 2013), con fechas de ca. 4.000 a.p.

\section{Discusión y Conclusiones}

Las clases artefactuales presentadas en este trabajo se ubican cronológicamente en distintos momentos del Holoceno temprano y medio. Para analizar y discutir el potencial tiempo-sensitivo de estos artefactos es importante no solo evaluar los cambios en la secuencia de los sitios Alero Cuevas sino la comparación macrorregional con otros sitios de la Puna argentina. La Figura 3 presenta la cronología de cada una de las clases registradas.

En la comparación macrorregional se observa que la clase 1 , conformada por las puntas triangulares apedunculadas de base recta, comprende un rango cronológico para la Puna argentina entre los 10.600 a.p. y los 8.500 a.p. Su dispersión es amplia, llegando a registrarse en el norte de Chile hasta los 10.800 a.p. (Núñez et al. 2005). De hecho, esta clase de puntas también se ha denominado Tuina por su hallazgo en el sitio homónimo (Núñez 1992). Se puede sostener que su presencia en el área surandina se registra por más de 2.000 años, en los momentos más tempranos de las ocupaciones humanas. En consecuencia, se considera que es un indicador tiempo-sensitivo relevante a nivel macrorregional.

La forma base corresponde a lasca y es importante destacar que los especímenes provenientes del sitio Alero Cuevas, Inca Cueva 4 y Hornillos 2 comparten la presencia de un eje técnico variable en su ubicación respecto del eje morfológico (Hocsman et al. 2012; Hoguin 2014; Hoguin y Restifo 2012). A futuro se espera profundizar en estos aspectos (ver Hoguin 2014 y Herrera et al. 2015 para una discusión tecnológica).

Además, esta clase presenta algunas variaciones en el tamaño. Especialmente, se observa una mayor longitud promedio en las puntas de los sitios Quebrada Seca 3 y Huachichocana III comparado con las de Hornillos 2, Inca Cueva 4 y Alero Cuevas. Sin embargo, más allá de esta variabilidad métrica, no se distingue un patrón de variación latitudinal o espacial particular. Por lo tanto, dentro de los procesos de transmisión cultural en escalas amplias que permitieron la replicación de esta clase, también se evidencia cierta tolerancia a la variación métrica en la longitud. Para los casos de Inca Cueva 4 y Quebrada Seca 3 no debe soslayarse el rol de la reactivación en los especímenes (Hocsman et al. 2012).

La clase 2 se superpone con la anterior, aunque sus fechas más tempranas están en torno a los límites cronológicos inferiores del sitio Alero Cuevas (ca. 9.880 a.p.), mientras que sus límites superiores en la Puna argentina se encuentran en Hornillos 2 (ca. 8.200 a.p.). Por lo tanto, si bien las clases 1 y 2 se superponen por 1.500 años, esta última se detecta más tardíamente en el registro macrorregional, y puede considerarse como un indicador cronológico relativo (Hoguin 2014). La dispersión espacial de la clase 2 también es amplia, registrándose tanto en el norte de Chile como en diversos sectores de la Puna argentina (Hoguin 2014; Núñez et al. 2005). Las fechas de Alero Cuevas se solapan con algunas obtenidas para esta clase en sitios como Tambillo 1 y 2, en el norte de Chile (Núñez et al. 2005). 


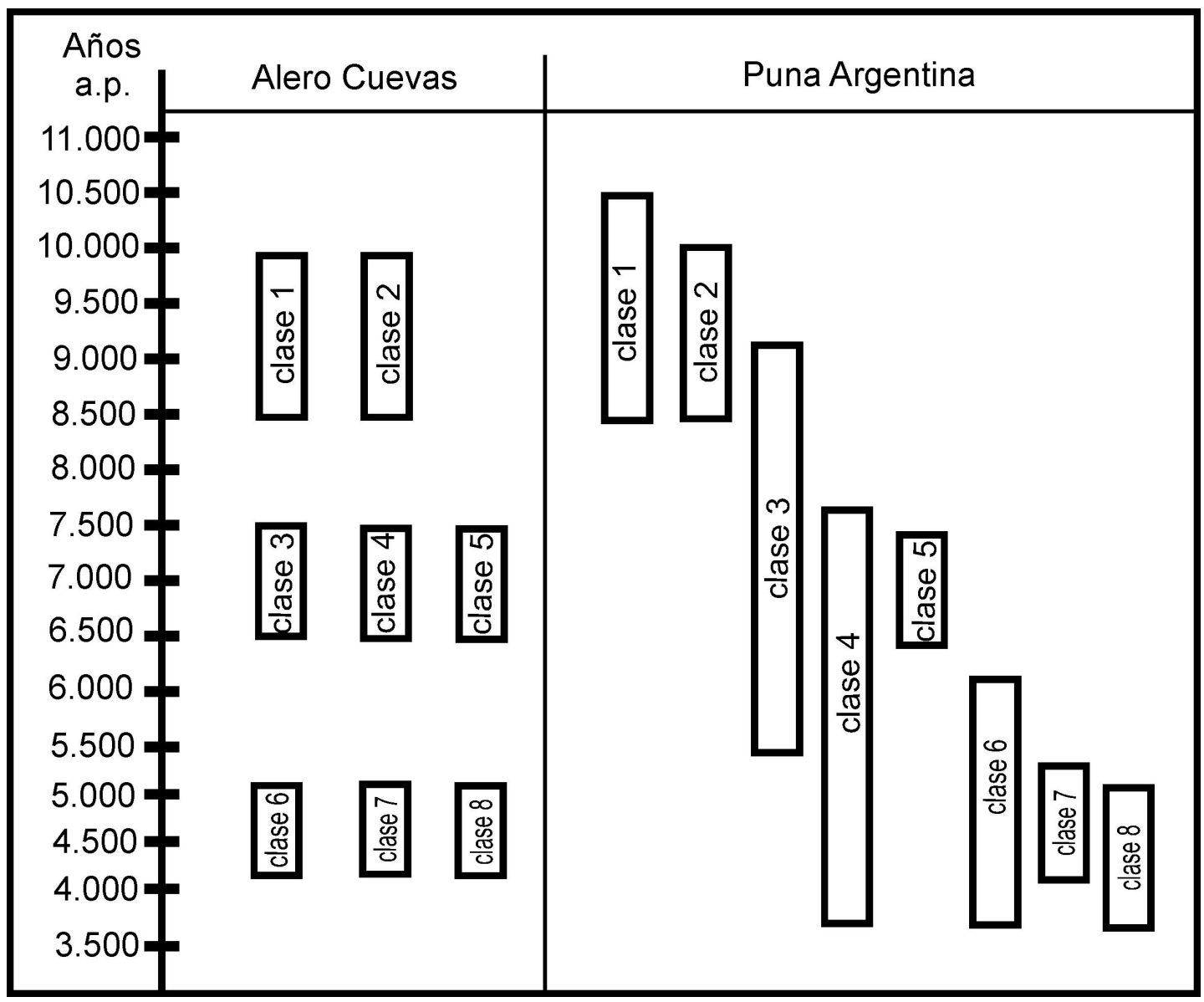

Figura 3. Clases de artefactos líticos y cronología comparada entre el sitio Alero Cuevas y la Puna argentina en general. Se considera la amplitud máxima de fechas de las clases registradas y en la columna de la Puna argentina se toman distintos sitios citados en el texto, incluyendo también a Alero Cuevas.

Lithic artefact classes and compared chronology between Alero Cuevas site and Puna of Argentina in general. We consider the maximum amplitude of the record dates of the classes, and in the Puna of Argentina column we take different sites mentioned in the text, including Alero Cuevas.

La clase 3 es más ubicua, dado que se encuentra distribuida en distintas partes del área andina. En otros contextos fue denominada como "Ayampitín" (ver Rivero 2008 para una discusión), pero su extensión temporal parece ser algo más amplia que otras clases analizadas. Si bien en Alero Cuevas se registra en la capa entre 7.500 y 6.500 a.p., también se menciona en Huachichocana III entre ca. 9.300 y 8.400 a.p., y en contextos posteriores (ca. 4.000 a.p.) (Hoguin 2014). Igualmente, la primera parte del Holoceno Medio (ca. 8.000-6.000 a.p.), coincidente con la cronología de la capa F3 de Alero Cuevas indicaría el momento de mayor proliferación de esta clase, tal como se evidencia en los sitios de la Puna de Jujuy y Catamarca (Hoguin 2014; Martínez 2003).
En la Puna de Salta, el sitio Abrigo Pozo Cavado, ubicado a $60 \mathrm{~km}$ al oeste del sitio Alero Cuevas, tiene ejemplares similares (López 2013). Asimismo, se debe considerar la variabilidad macrorregional, registrada tanto en características morfológicas como métricas. Este es el caso de los ejemplares de mayor tamaño (p.ej. Peñas de la Cruz A), que llegan a los 100 mm de longitud (Martínez 2003), mientras que el espécimen entero del sitio Alero Cuevas presenta una longitud menor (Tabla 2).

La clase 4 podría corresponderse con el tipo Punta de la Peña B, distinguido en la Puna de Catamarca (Hocsman 2006) lo cual extendería su cronología hasta fines del Holoceno Medio. Particularmente el ejemplar del sitio Alero Cuevas es 
similar al registrado en Punta de la Peña 4 (Hocsman 2006:259). Sin embargo, al tratarse de un espécimen posiblemente reactivado la comparación es difícil. En consecuencia, por el momento, si bien el potencial tiempo-sensitivo podría ser considerable, debe tomarse con precaución hasta no obtener nueva muestra en el sitio Alero Cuevas.

La clase 5 se ubica cronológicamente en ca. 7.500 a.p. en el sitio Alero Cuevas, y hasta el momento no pudo ser fechada en otros contextos a nivel macrorregional. De todas maneras, el aporte cronológico del sitio Alero Cuevas puede dar temporalidad relativa a algunos sitios con material de superficie donde esta clase es recurrente. Específicamente, Fernández (1971) la registró en la zona del Aguilar, en la Puna de Jujuy, Noroeste argentino. Si bien los ejemplares detectados por este autor presentan algunas variantes, se nota un patrón general a nivel morfológico. Por ejemplo, entre estas variantes se destaca la posibilidad de bordes con o sin denticulado y pedúnculos de base recta o convexa. La fecha obtenida para esta punta coincide con la idea de Fernández acerca de su asignación cronológica temprana. Por su parte, en la Cueva Salamanca 1, Puna de Catamarca, Noroeste argentino, Pintar (2014:60) presenta un ejemplar procedente de la capa 2(2) cuyas características podrían relacionarse con esta clase de puntas. En particular, se observa una pieza fragmentada, de bordes denticulados, hombros y pedúnculo de bordes convergentes hacia una base convexa. Este ejemplar se encuentra en un nivel de ca. 6.250 años a.p. Asimismo, en este sector de la Puna, se ha definido el tipo morfológico Quebrada Seca F, el cual presenta limbo triangular, pedúnculo de bordes convergentes y base recta (Hocsman 2006). Estos ejemplares se registran en niveles con fechas entre 5.000 y 3.000 a.p. Babot et al. (2010), plantean que estos artefactos se encuentran "transformados" como consecuencia de reformatización, probablemente a partir de clases más tempranas similares a las de la zona del Aguilar en Jujuy. En este sentido, la fecha del espécimen de Alero Cuevas puede indicar un antecedente temprano de estas morfologías. Por lo tanto, la clase 5 en Alero Cuevas cuenta con potencial tiempo-sensitivo (comienzos del Holoceno Medio), aunque a futuro sería importante aumentar la comparabilidad de conjuntos en capa.

La clase 6 también es ubicua a lo largo de la Puna argentina. En el sitio Alero Cuevas se registró un solo espécimen, pero su cronología en ca. 5.100-4.200 a.p., coincide con la obtenida en otros sitios de la Puna argentina, aumentando su potencial como indicador cronológico relativo. Este es el caso de sitios como Inca Cueva 7 y Abrigo Pozo Cavado (Aschero y Yacobaccio 1999; López 2013). También es recurrente en esta cronología en sitios del norte de Chile como Tulán 52 (De Souza et al. 2010). Sin embargo, también se registra más tempranamente en sitios como Hornillos 2 (ca. 6.300 a.p.) (Hoguin 2014).

La clase 7 no solo es importante por su alta representación en el sitio Alero Cuevas (comparable con las puntas triangulares apedunculadas de base recta), sino por asociarse con el cambio hacia forma base de hojas. Esta clase, presenta amplia distribución en la Puna de Jujuy y Salta, pero está prácticamente ausente en la Puna de Catamarca. Esto se relaciona con la ausencia de hojas en esta última región (Aschero y Hocsman 2011). Las primeras menciones acerca de esta clase de artefactos corresponden a los trabajos de Boman (1908) y Nordenskjöld (1903), provenientes del sitio Saladillo, Puna de Jujuy. En la década de 1950, se retoman a partir de los trabajos de González (1952) y Menghin (1954). Sin embargo, recién a principios de la década de 1960, Cigliano (1962) relocaliza el sitio y caracteriza a la "industria saladillense" a partir de talla unifacial. Los trabajos posteriores de Fernández (1971, 1983), presentan nueva información sobre estos artefactos, especialmente a través de la excavación del sitio Río Grande, Puna de Jujuy, fechado en ca. 5.500 a.p. Este fechado coincide con los obtenidos para esta clase artefactual en el sitio Ramadas Perfil Norte ubicado a $60 \mathrm{~km}$ al norte de Alero Cuevas, también en la Puna de Salta, en ca. 5.200 a.p. (Muscio 2004).

Su alta frecuencia en la capa F2 del sitio Alero Cuevas entre 5.100 y 4.200 a.p., y su ausencia en otras capas, permite sostener un alto potencial tiempo-sensitivo para esta clase. Esta hipótesis se refuerza al comparar macrorregionalmente y detectar su presencia en el tramo final del Holoceno Medio (5.500 a 4.000 a.p.), indicando un lapso de al menos 1.500 años de persistencia temporal. Al respecto, si bien su aparición podría ser anterior, los fechados obtenidos en el sitio Alero Cuevas no apoyan la idea de Fernández (1983) acerca de una "etapa decadente" hacia 6.000-5.000 a.p. Por el contrario, se considera aquí que este lapso abarca parte del momento de máxima proliferación de los "artefactos lanceolados unifaciales" o "Saladillo" 
en la Puna, mientras que la declinación de su frecuencia se registra después de ca. 4.000 a.p. Este lapso también podría relacionarse con el momento de mayor expansión de la forma base de hojas, ante la necesidad de aumentar la eficiencia en contextos de mayor demanda energética (López y Restifo 2012). En esta cronología se evidencian cambios relacionados con procesos de intensificación y domesticación de camélidos tanto en el sitio Alero Cuevas como a nivel macrorregional (López y Restifo 2012; Yacobaccio 2001).

En este contexto, se propone como hipótesis que la obtención de formas base relativamente estandarizadas que facilitan el reemplazo en caso de fractura o agotamiento, y una morfología elongada con filos largos de alto potencial de uso habría sido importante para cumplir distintas funciones, especialmente de procesamiento de recursos como los camélidos (López y Restifo 2012). Esto se diferencia de la idea de Fernández (1983) de considerar a estos artefactos como puntas. Si bien no se descarta esta función potencial en algunos especímenes, se considera que no habría sido la principal. De todas maneras, se espera que estudios funcionales de microdesgaste permitan avanzar en esta problemática a futuro.

A nivel métrico se evidencia una baja variación regional cuando se comparan los especímenes de Ramadas Perfil Norte, Río Grande y Alero Cuevas. Esto podría evidenciar procesos de transmisión cultural con mayor control de la variación, es decir una replicación de mayor fidelidad a nivel macrorregional (López y Restifo 2012). Desde una perspectiva evolutiva darwiniana, se espera que en contextos de mayor presión poblacional sobre los recursos, también aumenten los mecanismos de aprendizaje social que dan lugar a una baja variación dentro de cada clase (Bettinger y Eerkens 1999; Boyd y Richerson 1985).

Finalmente, la clase 8 tiene escasos correlatos en otras áreas. En la Puna de Catamarca, podría corresponderse con el tipo Peñas Chicas F (Hocsman 2006). En caso de ser correcta la comparación, esta clase se ubicaría a nivel cronológico hacia los 5.000-3.500 a.p. Esta cronología es coincidente con las fechas de la capa F2 del sitio Alero Cuevas. Esto refuerza la consideración de esta clase como indicador tiempo-sensitivo, pero deben obtenerse nuevos especímenes.

Para concluir, es importante resaltar que en el sitio Alero Cuevas están representadas distintas clases que pueden considerarse como indicadores cronológicamente diagnósticos. Entre ellas se destacan las clases 1 y 7 , por su alta representación en este sitio y su comparabilidad macrorregional (Figura 3). Si bien la secuencia multicomponente del sitio Alero Cuevas contribuye a analizar y discutir la persistencia temporal y el cambio de distintas clases artefactuales, solo la comparación macrorregional (p.ej. la Puna argentina) permite una contextualización de estos datos. De esta forma, se puede entender la relevancia de determinados artefactos para abordar cuestiones cronológicas, especialmente de contextos sin fechados absolutos, y el cambio en escalas de largo plazo.

La comparación macrorregional permitió establecer la presencia de clases con potencial tiempo-sensitivo, pero también la ausencia de otras en el sitio Alero Cuevas. Esto lleva a plantearse nuevos interrogantes sobre su variabilidad en escala cronológica y espacial amplia. En especial, es necesario preguntarse por qué en determinados momentos aumentan o disminuyen. Se plantea aquí que la acción de mecanismos de transmisión cultural en relación con los cambios en el ambiente socioecológico tiene mucho que explicar al respecto (López y Restifo 2012). Sin embargo, la interpretación de estos procesos excede los objetivos de este trabajo. Puntualmente, el análisis de las continuidades o discontinuidades en las clases de artefactos líticos tiempo-sensitivos aporta en esta dirección. El avance de la investigación y los estudios a futuro permitirán profundizar en los interrogantes señalados.

Agradecimientos: Al CONICET y todos los colaboradores en nuestro estudio. Además agradecemos a los editores y evaluadores que ayudaron a mejorar el trabajo. Nuestro agradecimiento al Dr. Rodolphe Hoguin por una lectura previa de este artículo. 


\section{Referencias Citadas}

Aschero, C. 1975. Ensayo para una clasificación morfológica de artefactos líticos. Informe de avance presentado al CONICET. Manuscrito en posesión del autor.

Aschero, C. 1983. Ensayo para una clasificación morfológica de artefactos líticos. Cátedra de ergología y tecnología, Universidad de Buenos Aires, Argentina.

Aschero, C. 1984. El sitio Inca cueva 4: Un asentamiento precerámico en la Quebrada de Inca Cueva (Jujuy, Argentina). Estudios Atacameños 7:62-72.

Aschero, C. y H.D. Yacobaccio 1999. 20 años después: Inca Cueva 7 reinterpretado. Cuadernos del Instituto Nacional de Antropología y Pensamiento Latinoamericano 18:7-18.

Aschero, C. y J. Martínez 2001. Técnicas de caza en Antofagasta de la Sierra, Puna Meridional Argentina. Relaciones de la Sociedad Argentina de Antropología 26:215-241.

Aschero, C. y S. Hocsman 2011. Arqueología de las ocupaciones cazadoras-recolectoras de fines del Holoceno medio de Antofagasta de la Sierra (Puna Meridional Argentina). Chungara Revista de Antropología Chilena 43:393-411.

Aschero, C., S. Hocsman y N. Ratto 2011. Las puntas de proyectil en "mandorla" de Inca Cueva 7: Caracterización tipológica e historia de vida (Puna de Jujuy, Argentina). Estudios Atacameños 41:5-28.

Babot, P., R. Cattáneo y S. Hocsman 2010. ¿Puntas de proyectil o cuchillos? Múltiples técnicas analíticas para una caracterización funcional de artefactos arqueológichos. En $\mathrm{La}$ Arqueometría en Argentina y Latinoamérica, editado por S. Bertolino, G.R. Cattáneo y A. Izeta, pp. 127-134. Editorial de la Facultad de Filosofía y Humanidades, Universidad Nacional de Córdoba, Córdoba.

Bar-Yosef, O. y S. Kuhn 1999. The big deal about blades: Lamina technologies and human evolution. American Anthropologist 101:322-338.

Boman, E. 1908. Antiquités de la Région Andine de République Argentine et du Desert D'Atacama. Impremerie National, París.

Bettinger, R. y J. Eerkens 1999. Point typologies, cultural transmission, and the spread of bow and arrow technology in the prehistoric Great Basin. American Antiquity 64:231-242.

Boyd, R. y P. Richerson 1985. Culture and the Evolutionary Process. University of Chicago Press, Chicago.

Capriles, J.M., S. Calla Maldonado y J. Albarracín-Jordán 2011. Tecnología lítica y estrategias de subsistencia durante los períodos Arcaico y Formativo en el Altiplano Central, Bolivia. Chungara Revista de Antropología Chilena 43:455-468.

Cigliano, E. 1962. Industrias precerámicas de la Puna Argentina. Ampurias 24:1-34

Clarckson, C. 2005. Tenuous types: Scraper reduction continuums in the eastern Victoria River Region, Northern Territory. En Lithics "Down Under", editado por C. Clarkson y S. Lamb, pp. 21-32. BAR International Series 1408, Oxford.

De Souza, P. 2004. Tecnologías de proyectil durante los períodos Arcaico y Formativo en el Loa superior (Norte de Chile): A partir del análisis de puntas líticas. Chungara Revista de Antropología Chilena 36:61-76.

De Souza, P., I. Cartajena, L. Núñez y C. Carrasco 2010. Cazadores-recolectores del Arcaico Tardío y desarrollo de complejidad social en la puna de Atacama: Las evidencias del sitio Tulán-52 (norte árido de Chile). Werkén 13:91-118.

Dunnell, R. 1977. Prehistoria Moderna. Introducción Sistemática al Estudio de la Arqueología Prehistórica. Ediciones Istmo, Madrid.

Fernández, J. 1971. La Edad de la Piedra en la Puna de Atacama (una investigación regional y cronológica, una aportación de la ciencia geográfica a la solución del problema vinculado a la temprana instalación humana en Sudamérica). Revista del Instituto de Antropología, serie 3, Tucumán.

Fernández, J. 1983. Río Grande. Exploración de un centro precerámico en las altas montañas de Jujuy, Argentina. Ampurias 45/46:54-83.

Fernández Distel, A. 1974. Excavaciones arqueológicas en las Cuevas de Huachichocana, Departamento de Tumbaya, Pcia. de Jujuy. Relaciones de la Sociedad Argentina de Antropología VIII:101-127.

González, A.R. 1952. Antiguo horizonte precerámico en las Sierras Centrales de Argentina. Runa V:110-133.

Herrera, K., P. Ugalde, D. Osorio, J. Capriles, S. Hocsman y C. Santoro 2015. Análisis tecno-tipológico de instrumentos líticos del sitio Arcaico temprano Ipilla 2 en los Andes de Arica, Chile. Chungara Revista de Antropología Chilena 47:41-52.

Hocsman, S. 2006. Producción Lítica, Variabilidad y Cambio en Antofagasta de la Sierra ca. 5500-1500 AP. Tesis para optar al grado de doctor. Facultad de Ciencias naturales y Museo, Universidad Nacional de La Plata, La Plata.

Hocsman, S., J.G. Martínez, C. Aschero y A. Calisaya 2012. Variability of triangular non-stemmed projectile points of early hunter-gatherers of the Argentinean Puna. Current Research in the Pleistocene (Special Edition):63-68.

Hoguin, R. 2014. Secuencia cronológica y tecnología lítica en la Puna Seca y Salada de los Andes Centro-Sur para el Holoceno temprano y medio a través del ejemplo de Susques. Relaciones de la Sociedad Argentina de Antropología XXXIX (2):333-364.

Hoguin, R. y F. Restifo 2012. Cultural transmission patterns in projectile points manufacture: Implications for the Early settlement of the Argentinean Puna. Current Research in the Pleistocene (Special Edition): 69-74.

Hoguin, R. y H. Yacobaccio 2012. Análisis lítico de ocupaciones del Holoceno Medio de Hornillos 2 (Jujuy, Argentina): discutiendo la tecnología y distribución de las puntas de proyectil "San Martín”. Chungara Revista de Antropología Chilena 44:85-99.

Jones, G. y C. Beck 1992. Chronological Resolution in Distributional Archaeology. En Space, Time and Archaeological Landcapes, editado por J. Rossignol y L. Wandsnider, pp. 167192. Springer, New York.

Klink, C.J. y M. Aldenderfer 2005. A projectile point chronology for the South-Central Andean Highlands. En Advances in Titicaca 
Basin Archaeology, vol. 1, editado por C. Stanish, A.B. Cohen y M. Aldenderfer, pp. 25-54. Cotsen Institute of Archaeology, University of California, Los Angeles.

López, G. 2008. Arqueología de Cazadores y Pastores en Tierras Altas. Ocupaciones Humanas a lo Largo del Holoceno en Pastos Grandes, Puna de Salta, Argentina. BAR International Series 1854, South American Archaeology Series No. 4, Oxford.

López, G. 2013. Ocupaciones humanas y cambio a lo largo del Holoceno en abrigos rocosos de la Puna de Salta, Argentina: Una perspectiva regional. Chungara Revista de Antropología Chilena 45:411-426.

López, G. y F. Restifo 2012. The Middle Holocene domestication and intensification of camelids in north Argentina, tracked by zooarchaeology and lithics. Antiquity 86:1041-1054.

López, G. y F. Restifo 2014. Procesos de diversificación, intensificación y domesticación durante el Holoceno en las tierras altas del norte de Argentina y Chile: Aportes desde la Puna de Salta. Comechingonia 18:95-116.

Martínez, J.G. 2003. Ocupaciones Humanas Tempranas y Tecnología de Caza en la Microrregión de Antofagasta de la Sierra (10000-7000 AP). Tesis para optar al grado de doctor. Facultad de Ciencias Naturales e Instituto Miguel Lillo, Universidad Nacional de Tucumán, Tucumán.

Méndez, C., K. Rademaker y V. Standen 2015. Early lithic technology in South America: Moving beyond regional projectile points typologies. Chungara Revista de Antropología Chilena 47:3-5.

Menghin, O. 1954. Culturas precerámicas en Bolivia. Runa VI:125-132.

Morales, M. 2011. Arqueología Ambiental del Holoceno Temprano y Medio en la Puna Seca Argentina. Modelos Paleoambientales Multiescalas y sus Implicancias Para la Arqueología de CazadoresRecolectores. BAR International series, Oxford.

Moreno, E. 2011. Tecnología de caza en Antofalla, Departamento de Antofagasta de la Sierra, Catamarca. Revista del Museo de Antropología 4:17-32.

Muscio, H.J. 2004. Dinámica Poblacional y Evolución durante el Período Agroalfarero Temprano en el Valle de San Antonio de los Cobres, Puna de Salta, Argentina. Tesis para optar al grado de doctor en Ciencias Antropológicas. Facultad de Filosofía y Letras, Universidad de Buenos Aires, Buenos Aires.

Nelson, M. 1991. The study of technological organization. Archaeological Method and Theory 3:57-100.

Nordensköld, E. 1903. Einiges über das gebiet, wo sich Chaco und Andean begegnen. Globus LXXXIV:197.

Núñez, L. 1992. Ocupación arcaica en la Puna de Atacama: secuencia, movilidad y cambio. En Prehistoria Sudamericana.
Nuevas Perspectivas, editado por B. Meggers, pp. 283-307. Taraxacum, Washington.

Núñez, L., M. Grosjean e I. Cartejena 2005. Ocupaciones Humanas y Paleoambientes en la Puna de Atacama. Universidad Católica del Norte -Taraxacum, San Pedro de Atacama.

O’Brien, M. y L. Lyman 2000. Applyng Evolutionary Archaeology. A Systematic Approach. Kluwer Academic Publishers, Nueva York.

O'Brien, M. y L. Lyman 2002. The epistemological nature of archaeological units. Anthropological Theory 2:37-56.

Patané Aráoz, J. 2013. Prospecciones arqueológicas en Salinas Grandes (Departamento La Poma, Provincia de Salta) y reporte de una punta "cola de pescado". Relaciones de la Sociedad Argentina de Antropología XXXVIII (1):247-255.

Pintar, E. 2014. Continuidades e hiatos ocupacionales durante el Holoceno Medio en el borde oriental de la Puna Salada, Antofagasta de la Sierra, Argentina. Chungara Revista de Antropología Chilena 46:51-71.

Ratto, N. 2006. El Arcaico y el Formativo en la Puna de Chaschuil a través del diseño de las puntas líticas (Departamento Tinogasta, Catamarca). Cazadores Recolectores del Cono Sur 1:93-110.

Restifo, F. 2013a. Tecnología Lítica en la Puna de la Provincia de Salta a lo Largo del Holoceno Temprano y Medio: Patrones de Variación y Procesos de Cambio. Tesis para optar al grado de doctor en Ciencias Antropológicas. Facultad de Filosofía y Letras, Universidad de Buenos Aires, Buenos Aires.

Restifo, F. 2013b. Tecnología de caza durante el Holoceno temprano y medio en la Puna de la Provincia de Salta (República Argentina): Patrones de variación y procesos de cambio. Comechingonia 17:59-84.

Restifo, F. 2015. Tecnología de hojas líticas en tierras altas andinas: Perspectivas desde la Puna de la Provincia de Salta (Argentina). Estudios Atacameños 51:33-51.

Rivero, D. 2008. Alberto Rex González y la vigencia de sus aportes a la arqueología de cazadores-recolectores de Argentina. Comechingonia Virtual 4:226-234.

Schobinger, J. 1988. Prehistoria de Sudamérica. Culturas Precerámicas. Alianza Editorial, Barcelona.

Yacobaccio, H.D. 2001. La domesticación de camélidos en el Noroeste Argentino. En Historia Argentina Prehispánica, Tomo 1, editado por E.E. Berberián y A.E. Nielsen, pp. 7-40. Editorial Brujas, Córdoba.

Yacobaccio, H.D. y V. Vilá 2002. Condiciones mecanismos y consecuencias de la domesticación de camélidos. Estudios Sociales del NOA 3:4-27.

Yacobaccio, H.D. y M. Morales 2011. Ambientes pleistocénicos y ocupación humana temprana en la Puna Argentina. Boletín de arqueología PUCP 15:337-356. 
